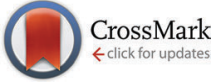

Cite this: Phys. Chem. Chem. Phys. 2016, 18, 11839

Received 28th January 2016,

Accepted 1st February 2016

DOI: $10.1039 / \mathrm{c} 6 \mathrm{cp} 00636 \mathrm{a}$

www.rsc.org/pccp

\section{An electronic aromaticity index for large rings}

\author{
Eduard Matito abc
}

We introduce a new electronic aromaticity index, AV1245, consisting of an average of the 4-center multicenter indices $(\mathrm{MCl})$ along the ring that keeps a positional relationship of 1, 2, 4, 5. AV1245 measures the extent of transferability of the delocalized electrons between bonds $1-2$ and $4-5$, which is expected to be large in conjugated circuits and, therefore, in aromatic molecules. A new algorithm for the calculation of $\mathrm{MCl}$ for large rings is also introduced and used to produce the data for the calibration of the new aromaticity index. AV1245 does not rely on reference values, does not suffer from large numerical precision errors, and it does not present any limitation on the nature of atoms, the molecular geometry or the level of calculation. It is a size-extensive measure with low computational cost that grows linearly with the number of ring members. Therefore, it is especially suitable to study the aromaticity of large molecular rings such as those occurring in belt-shaped Möbius structures or porphyrins. The analysis of AV1245 in free-base and bis-metalated Pd [32] octaphyrins $(1,0,1,0,1,0,1,0)$ completes this study.

\section{Introduction}

Chemistry is essentially an experimental science that evolved through experimentation and it has been built upon a series of empirically proved laws and models. On the other side, quantum mechanics relies on postulates from which a solid theory has been constructed. Both focus on the study of matter, however, quantum mechanics can anticipate the electronic structure of matter and it could, in principle, replace the laws and models of chemistry by physically sound theories. Notwithstanding, after many years of the advent of quantum mechanics, several chemical concepts with high predictable power still prevail. Most of these concepts have not found (and most likely cannot find) a solid root in quantum theory because there is no observable behind them. One finds many such concepts in the literature (e.g., chemical bonding, bond order, ionicity, electron population, agostic bond, etc.) that are still widely used to predict or explain the electronic structure of molecules or reaction mechanisms. ${ }^{1}$ One of the most employed terms in the literature - and one of the most controversial ${ }^{2}$ ones - is aromaticity. ${ }^{3-7}$ Aromaticity is associated with cyclic electron delocalization in closed circuits that gives rise to energy stabilization, bond length equalization, large magnetic anisotropies and abnormal chemical shifts, among other effects. Many of these aromaticity manifestations

\footnotetext{
${ }^{a}$ Faculty of Chemistry, University of the Basque Country UPV/EHU, and Donostia International Physics Center (DIPC), P.K. 1072, 20080 Donostia, Euskadi, Spain. E-mail: ematito@gmail.com

${ }^{b}$ IKERBASQUE, Basque Foundation for Science, 48011 Bilbao, Spain

${ }^{c}$ Instituto de Quimica at the Universidad Autónoma de México (UNAM), Mexico
}

can be measured in appropriate quantities, the aromaticity indices, that allow for aromaticity scales. As a result, nowadays there are a number of indices available in the literature, often offering disparate results about the aromaticity of certain chemical species. ${ }^{8}$ The discovery of new aromatic species ${ }^{9-11}$ that extend well beyond the realm of organic chemistry has challenged our understanding of aromaticity and it has put forward the limitations of existing aromaticity indices to deal with these new chemical creatures. ${ }^{12}$ We have contributed to calibrate and test the limits of applicability of these measures by designing a series of tests that aromaticity indices should pass. ${ }^{13,14}$ The indices based on multicenter electron sharing were the most successful ones. These indices are free of arbitrary references, can be applied to organic and inorganic species alike and, their latest versions are free of the ring-size dependency of the original definitions. ${ }^{15}$ Despite these benefits, the multicenter indices suffer from a series of problems that prevent their application in large rings. Among molecules with large rings we find porphyrinic systems, which are involved in new interesting problems such as the aromaticity of (expanded) ${ }^{16}$ porphyrins ${ }^{17,18}$ and its relationship with the UV absorption spectra or Hückel-to-Möbius topological switches. ${ }^{19-21}$ Porphyrins are ubiquitous pigments in nature, playing prominent roles in several biological processes as well as the preparation of photovoltaic cells, nonlinear optical materials and electroluminescene displays. ${ }^{22}$ Expanded porphyrins are also versatile compounds giving rise to a plethora of twisted Möbius aromatic structures that can be utilized in a number of applications. ${ }^{17}$ The salient properties of porphyrins and expanded porphyrins are related to their long conjugated $\pi$-electron closed circuits that provide these molecules a certain aromatic character. The presence of conjugated circuits in 
these large molecular rings calls for aromaticity measures that can be unambiguously used in rings of arbitrary size and do not suffer from severe limitations. Thus far, these rings have been studied by FLU $^{13}$ (see Section 2), the nuclear independent chemical shifts $(\mathrm{NICS})^{21}$ or the harmonic oscillator model of aromaticity (HOMA). ${ }^{20}$ $\mathrm{FLU}^{23}$ and $\mathrm{HOMA}^{24}$ measure the dissimilarity of the target molecules with some reference aromatic molecules and, therefore, they cannot be used to study reactivity. ${ }^{25} \mathrm{NICS}^{5,26}$ is affected by the presence of the magnetic field of neighboring atoms, such as the metals coordinated in metalated porphyrins. ${ }^{27}$

The goal of this paper is introducing a new electronic aromaticity index that can be applied to rings of arbitrary size. The work is organized as follows: first, we will review the expressions for electron-delocalization aromaticity indices and reveal their strengths and weaknesses; second, we will describe an algorithm that permits us to calculate the multicenter index (MCI) on larger rings but it is still limited to rings of medium size; then we will provide the expression of a new multicenter index that will be finally analyzed in a series of compounds to assess its performance.

\section{Two-center indices}

In the following we will indicate the coordinates of the electron using the short-hand notation $1 \equiv\left(\vec{r}_{1}, \sigma_{1}\right)$ and $d_{1} \equiv \mathrm{d} \vec{r}_{1} \mathrm{~d} \sigma_{1}$ for the derivatives. Unless otherwise indicated, we will assume a wavefunction constructed from a single-determinant, for a more general approach we suggest ref. 28. We will measure the aromaticity in a ring structure that consists of $n$ atoms, represented by the string $\mathscr{A}=\left\{A_{1}, A_{2}, \ldots, A_{n}\right\}$, whose elements are ordered according to the connectivity of the atoms in the ring.

The delocalization index or the electron sharing index (ESI) ${ }^{29-31}$ is a measure of covariance between populations in atoms $A$ and $B^{32}$ and it is defined as

$$
\delta(A, B)=\int_{A} \int_{B} d_{\mathbf{1}} d_{\mathbf{2}}\left[\rho(\mathbf{1}) \rho(\mathbf{2})-\rho_{2}(\mathbf{1}, \mathbf{2})\right]=2\left\langle\left(\bar{N}_{A}-\hat{N}_{A}\right)\left(\bar{N}_{B}-\hat{N}_{B}\right)\right\rangle
$$

with $\hat{N}_{A}$ being the particle operator applied to atom $A$ and $\bar{N}_{A}$ the average number of electrons in $A$,

$$
\bar{N}(A)=\int \hat{N}_{A} \rho(\mathbf{1}) d_{\mathbf{1}} \equiv \int_{A} \rho(\mathbf{1}) d_{\mathbf{1}}
$$

and where $\rho(\mathbf{1})$ is the electron density and $\rho_{2}(\mathbf{1}, 2)$ the pair density. ${ }^{33}$ This quantity is commonly used as a measure of the number of electron pairs shared between two atoms and, therefore, regarded as a covalency index or as a bond order.

The ESI of two contiguous atoms in the ring of an aromatic molecule shows values intermediate between single and double bond character. However, this value oscillates depending on the two atoms bonded. The aromatic fluctuation index (FLU) compares the ESI for bond pairs along the ring with typical aromatic bond pairs: ${ }^{23,34}$

$$
\operatorname{FLU}(\mathscr{A})=\frac{1}{n} \sum_{i=1}^{n}\left[\left(\frac{V\left(A_{i}\right)}{V\left(A_{i-1}\right)}\right)^{\alpha}\left(\frac{\delta\left(A_{i}, A_{i-1}\right)-\delta_{\mathrm{ref}}\left(A_{i}, A_{i-1}\right)}{\delta_{\mathrm{ref}}\left(A_{i}, A_{i-1}\right)}\right)\right]^{2}
$$

where $A_{0} \equiv A_{n}$ and $V(A)$ is the atomic valence that for a closedshell system reads ${ }^{35}$

$$
V(A) \equiv \delta(A)=\sum_{B \neq A} \delta(A, B)
$$

and $\alpha$ is a simple function to ensure that the first term in eqn (3) is always greater or equal to 1 ,

$$
\alpha= \begin{cases}1 & V\left(A_{i-1}\right) \leq V\left(A_{i}\right) \\ -1 & V\left(A_{i}\right)<V\left(A_{i-1}\right)\end{cases}
$$

$\delta_{\text {ref }}(A, B)$ is taken from an aromatic molecule which has the pattern of bonding $A-B$. In the present case, where only $\mathrm{C}-\mathrm{C}$ and $\mathrm{C}-\mathrm{N}$ bonds will be taken into account, the molecules chosen as an aromatic reference are benzene $(\mathrm{C}-\mathrm{C})$ and pyridine $(\mathrm{C}-\mathrm{N})$. FLU is close to zero in aromatic species, and greater than zero for non-aromatic or antiaromatic species.

The aromaticity indices based on references do not actually measure aromaticity but they measure the dissimilarity with respect to some aromatic molecules. In this sense, these indices (HOMA and FLU) are not adequate to describe reactivity. ${ }^{13,25}$ For instance, in the case of the Diels-Alder reaction they cannot recognize the transition state (TS) as the most aromatic point along the reaction path because the product, cyclohexene, is more similar to benzene than the TS, which shows large $\mathrm{C}-\mathrm{C}$ distances in the bonds to be formed. ${ }^{25}$

In 1993, Fulton ${ }^{36}$ unveiled an interesting feature of aromatic six-membered rings. These species show larger ESI values for two atoms in the para position than two atoms in the meta position despite the larger separation between the former pair. ${ }^{37}$ Poater and coworkers used this idea to define an aromaticity index for six-membered rings known as the para-related delocalization index (PDI): ${ }^{38}$

$$
\operatorname{PDI}(\mathscr{A})=\frac{\delta\left(A_{1}, A_{4}\right)+\delta\left(A_{2}, A_{5}\right)+\delta\left(A_{3}, A_{6}\right)}{3}
$$

The larger the PDI, the more aromatic the molecule. Both FLU and PDI rely on two-center measures of aromaticity and, therefore, they might fail in molecules where the aromaticity involves complicated multicenter conjugation patterns.

\section{Multicenter indices}

To our knowledge, Giambiagi was first to develop an index to measure multicenter bonding. ${ }^{39}$ The same expression applied to a molecular ring was denoted as $I_{\text {ring }}$ and used to account for aromaticity $^{40}$

$$
I_{\text {ring }}(\mathscr{A})=2^{n-1} \sum_{i_{1} i_{2} \ldots i_{n}} S_{i_{1} i_{2}}\left(A_{1}\right) \cdots S_{i_{n} i_{1}}\left(A_{n}\right)
$$

where $n_{i} \in[0,1]$ is the occupancy of the corresponding natural orbital and $S_{i j}\left(A_{1}\right)$ is the atomic overlap matrix (AOM) of atom $A_{1}$,

$$
S_{i j}\left(A_{1}\right)=\int_{A_{\mathbf{1}}} d_{\mathbf{1}} \phi_{i}^{*}(\mathbf{1}) \phi_{j}(\mathbf{1})
$$


with $\phi_{i}(\mathbf{1})$ being a natural orbital. $I_{\text {ring }}$ depends on the order of the atoms in the string for $n>3$.

Bultinck et al. ${ }^{41}$ suggested MCI, which is computed by summing up all the possible $I_{\text {ring }}$ contributions obtained from the permutations of the atoms in $\mathscr{A}$,

$$
\operatorname{MCI}(\mathscr{A})=\frac{1}{2 n} \sum_{\mathscr{P}(\mathscr{A})} I_{\text {ring }}(\mathscr{A})
$$

where $\mathscr{P}$ is an operator acting on $\mathscr{A}$ that produces all the $n !$ permutations of its atoms. The MCI is related to the $n$-center electron sharing index (nc-ESI), ${ }^{28,42-44} \delta(\mathscr{A})$, as

$$
\operatorname{MCI}(\mathscr{A})=\frac{(n-1) !}{2} \delta(\mathscr{A})=(-2)^{n-2}\left\langle\prod_{i=1}^{n}\left(\bar{N}_{A_{i}}-\hat{N}_{A_{i}}\right)\right\rangle
$$

Except for a scalar factor eqn (1) can be obtained as a particular case of the latter expression for $n=2$.

The r.h.s. of eqn (10) indicates that the $\operatorname{MCI}(\mathscr{A})$ is a measure of how the electron distribution of $n$ particles in $n$ atoms $A_{1}, \ldots, A_{n}$ is skewed from its mean and it is related to the simultaneous electron fluctuation among these atomic basins. Therefore, $I_{\text {ring }}$ measures the delocalization along the ring while MCI also accounts for the delocalization across the ring. In the author's opinion $I_{\text {ring }}$ should be connected to cyclic electron delocalization, which is the term used by Schleyer in the IUPAC to define aromaticity. ${ }^{3}$ On the other hand, the MCI measures the global electron delocalization in the ring that is obviously not limited to the cyclic electron delocalization along the ring structure. This distinction becomes important for small rings (typically fourand five-membered rings) where cross-contributions of electron delocalization might be more important. ${ }^{45}$

These expressions, eqn (7) and (9), suffer from ring-size dependency. Indeed, the overlaps entering eqn (7) are, in the absolute value, usually smaller than one and, therefore, the larger the ring the smaller the $I_{\text {ring }}$ and MCI values. A few years ago, we suggested a normalization that not only avoids this problem but also closely matches the classical topological resonances per $\pi$ electron (TREPE) ${ }^{46}$ of aromatic annulenes and their ions. ${ }^{15}$ In this paper, for the sake of simplicity, we just retain the normalization that produces ring-size-independent indices by taking the $1 / n$ power of eqn (7) and (9), i.e., $I_{\text {ring }}^{1 / n}$ and MCI ${ }^{1 / n}$. If $I_{\text {ring }}$ or MCI values are negative, we will take the power of the absolute value and multiply the resulting number by minus one.

\section{Multicenter index drawbacks}

\subsection{The atomic partition}

Different atomic partitions can be used to compute the overlaps in eqn (8); however, some partitions produce completely wrong results, such as indicating benzene as the least aromatic species of a large set of six-membered rings. ${ }^{47}$ This result is in contrast with other electronic aromaticity indices such as FLU, ${ }^{23,34}$ which is less sensitive to atomic partition ${ }^{48}$ (see also Table 1) because covalent bond orders do not vary much among atomic partitions. ${ }^{49}$ In this respect, the most suitable atomic partition ${ }^{32,47,50}$ explored thus far is Bader's quantum theory of atoms in
Table $1 \mathrm{MCl}$ and $I_{\text {ring }}$ values for benzene, cyclohexane and pyridine using QTAIM, Löwdin and Mulliken partitions along with cc-pVDZ (PF) and aug-

\begin{tabular}{|c|c|c|c|c|c|c|}
\hline & \multicolumn{2}{|c|}{ QTAIM } & \multicolumn{2}{|c|}{ Mulliken } & \multicolumn{2}{|c|}{ Löwdin } \\
\hline & $\mathrm{PF}$ & $\mathrm{DPF}$ & $\mathrm{PF}$ & DPF & $\mathrm{PF}$ & DPF \\
\hline \multicolumn{7}{|l|}{$I_{\text {ring }}$} \\
\hline Benzene & 0.049 & 0.047 & 0.077 & 0.482 & 0.053 & 0.004 \\
\hline Cyclohexane & 0.000 & 0.000 & 0.000 & 0.001 & 0.001 & 0.001 \\
\hline Pyridine & 0.046 & 0.045 & 0.076 & 0.041 & 0.054 & 0.005 \\
\hline \multicolumn{7}{|l|}{ MCI } \\
\hline Benzene & 0.073 & 0.071 & 0.115 & 0.759 & 0.080 & 0.020 \\
\hline Cyclohexane & 0.000 & 0.000 & 0.000 & 0.011 & 0.001 & 0.001 \\
\hline Pyridine & 0.068 & 0.066 & 0.112 & 0.254 & 0.082 & 0.021 \\
\hline \multicolumn{7}{|l|}{ FLU } \\
\hline Benzene & 0.000 & 0.000 & 0.000 & 0.000 & 0.000 & 0.000 \\
\hline Cyclohexane & 0.096 & 0.095 & 0.067 & 0.027 & 0.083 & 0.047 \\
\hline Pyridine & 0.004 & 0.005 & 0.000 & 0.568 & 0.000 & 0.004 \\
\hline \multicolumn{7}{|l|}{ PDI } \\
\hline Benzene & 0.103 & 0.103 & 0.085 & 0.308 & 0.106 & 0.155 \\
\hline Cyclohexane & 0.009 & 0.009 & -0.003 & 0.056 & 0.009 & 0.052 \\
\hline Pyridine & 0.103 & 0.103 & 0.085 & -0.034 & 0.110 & 0.156 \\
\hline
\end{tabular}
cc-pVDZ (DPF) basis sets

molecules (QTAIM) partition. ${ }^{51}$ Three-dimensional atomic partitions such as QTAIM carry some errors due to the fact that numerical integrations of the AOM are performed in non-regular three-dimensional atomic basins. In this regard, one could advocate for the use of Hilbert-space partitions that involve analytical integrations for atom-centered basis sets. However, it is well known that Hilbert-space-based population analyses suffer from spurious results when diffuse functions, lacking a prominent atomic character, are included in the basis sets. The results collected in Table 1 suggest that multicenter indices suffer to a greater extent from basis-set dependency than the two-center counterparts. Both $I_{\text {ring }}$ and MCI produce bogus results for basis sets containing diffuse functions if Mulliken or Löwdin partitions are used to define the atoms in the molecule. Mulliken results give unusually large values while Löwdin partition values are too small. The PDI (that measures two-center non-bonded electron sharing, see Section 2) is also largely affected by the inclusion of diffuse functions, whereas FLU, ${ }^{23}$ which is constructed from the 2c-ESIs between bonded atoms, only shows important changes for pyridine calculated by Mulliken's partition. Interestingly, for the cc-pVDZ basis set, the agreement between Löwdin and QTAIM partition is quite good for all the indices. It is thus apparent that Hilbert-space-based partitions can only be used in the absence of diffuse functions. Since many chemical systems exhibit highly delocalized electronic structures that call for the use of diffuse functions, we recommend the use of three-dimensional space partitions for the calculation of multicenter indices.

\subsection{Computational cost and numerical precision}

The operation $\mathscr{P}(\mathscr{A})$ in eqn (9) produces $n$ ! summations and thus constitutes the bottleneck of the MCI calculation. Overall, if carried out naively, the computational cost of MCI is $m^{3} n ! / 2$, where $m$ is the number of occupied orbitals and we have taken into account that there is only $(n-1) ! / 2$ distinct permutations for the elements in $\mathscr{A}$. 
The computation of three-dimensional atomic partitions carries numerical precision errors in the calculation of the AOMs and the total MCI error grows with the ring size. There are three effects as $\Delta n$ members are included in $\mathscr{A}$ : (i) on the one hand, the number of summations in eqn (9) increases by roughly a factor of $n^{\Delta n}$, (ii) each term in the summation of eqn (7) involves $\Delta n$ additional multiplications and (iii) the increase of the ring size probably leads to the increase of the dimension of the AOM, as larger molecules have more electrons and, therefore, more orbitals will be involved in the calculation. AOM elements are much smaller than the ones for the delocalized orbitals that contribute to the MCI and hence, as the ring size increases the error in each summation can easily increase beyond the machine precision. In addition, for calculations using correlated wavefunctions (see next section) this situation worsens considerably. For single-determinant methods (or Kohn-Sham density functional theory) the dimension of AOM is proportional to $N^{2}$, where $N$ is the number of electrons, but for correlated calculations the dimension is $m^{2}$, where $m$ is the number of occupied orbitals.

There are a number of tips we can follow to improve the computational performance and also minimize the error in the MCI. First, we should use very accurate numerical integration schemes to avoid large error propagations. Second, we should make a careful selection of the orbitals involved in the MCI calculation. Bultinck et al. introduced the pseudo- $\pi$ method, ${ }^{52,53}$ which reduces the size of $m$ by considering only the $\pi$ orbitals in the system. The approximation works very efficiently for polycyclic aromatic hydrocarbons but many large rings, such as those showing in belt-shaped Möbius molecules, are not planar and are thus not suitable for the classification of their orbitals into $\sigma$ and $\pi$ types. One can also manually select the set of orbitals that can contribute to the aromaticity of the system. Usually only valence orbitals are delocalized enough to contribute to the MCI and, therefore, the cost can be safely reduced by only including these orbitals. For highly symmetric molecules, the use of symmetry constraints can also reduce the cost by considering only symmetrically distinct permutations. Besides, the implementation of the Strassen algorithm for matrix multiplication can also contribute to decrease the cost to $m^{2.8} n ! / 2$ but it is only worth it for very large matrices $(m \gg 1000)$.

Overall, the large error propagation cannot be avoided and it is an inherent problem for the calculation of MCI and $I_{\text {ring; }}$; fortunately, these problems only occur for large rings $(n \geq 10)$.

\subsection{Correlated wavefunctions}

The calculation of the MCI from a correlated wavefunction through eqn (10) bears high computational cost, associated with the computation of the $n$-density. The calculation of $n$-order densities using correlated wavefunctions for $n>3$ is typically beyond the capabilities of current computational resources, except for very small molecules. For this reason, many authors, ${ }^{54}$ including ourselves, ${ }^{15}$ have decided to approximate the $n$-order densities that provide cost-efficient MCI calculations using correlated wavefunctions. Lately, we have introduced two approximations of $n \mathrm{c}$-ESI that can be shown in the following expression for the MCI:

$$
\operatorname{MCI}(\mathscr{A})=\frac{2^{(n-2)}}{n} \sum_{\mathscr{P}(\mathscr{A})} \sum_{i_{1} \ldots i_{n}}\left(n_{i_{1}} \cdots n_{i_{n}}\right)^{a} S_{i_{1} i_{2}}\left(A_{1}\right) \cdots S_{i_{n} i_{1}}\left(A_{n}\right)
$$

where $a$ is a constant and $\left\{n_{i}\right\}$ are natural orbital occupancies. $a=1$ corresponds to the single-determinant expression of the $n$-densities, whereas $a=1 / 2$ and $a=1 / 3$ are our proposals. The latter has been shown to provide the most accurate results for 3c-ESIs, even better than more sophisticated 3-density approximations. ${ }^{28}$ However, the exact calculations of $3 \mathrm{c}$-ESIs tend to provide very small values and certain capabilities of the $3 \mathrm{c}$-ESIs are lost upon inclusion of electron correlation. ${ }^{28}$ For instance, eqn (11) with $a=1$ is the only variant of MCI calculation that permits us to distinguish between $3 \mathrm{c}-2 \mathrm{e}$ and $3 \mathrm{c}-4 \mathrm{e}$ interactions. ${ }^{42,55}$ For this reason, in recent aromaticity studies using correlated wavefunctions we have preferred the $a=1$ approximation, which provides more sensible numbers. ${ }^{15,56}$

\section{New algorithm for the $\mathrm{MCl}$}

In order to bring down the computational cost it is much more profitable to reduce the number of distinct permutations rather than reducing the scaling of the number of basis functions. To this aim, we devise an algorithm that screens the superatomic overlap matrices (SAOMs hereafter) that result from the partial summation of some indices, for example,

$$
\begin{aligned}
S_{i j}(A B) & =\sum_{k} S_{i k}(A) S_{k j}(B) S_{i j}(A B C)=\sum_{k l} S_{i k}(A) S_{k l}(B) S_{l j}(C) \\
& =\sum_{l} S_{i l}(A B) S_{l j}(C) S_{i j}(A B C D)=\sum_{k} S_{i k}(A B) S_{k j}(B C)
\end{aligned}
$$

These matrices can be precomputed only for the SAOMs that produce some significant (above some threshold) value. The decrease of the number of SAOMs lowers the cost of the MCI calculation by reducing the number of possible permutations. The most efficient way to construct these matrices is by successive duplication of the SAOM order following the binary representation of the number of atoms in the ring $(n)$. First of all, we construct a supermatrix of order $p$, where $p$ is the largest number $2^{p}$ that is smaller or equal to $n$, by consecutive self-combination of lower-order matrices. Namely, we first construct the SAOMs of order 2 (2-SAOMs) from the AOMs of the atoms in our ring and the 2-SAOMs are combined among themselves to build the 4 -SAOMs and so on until $2^{p}$-SAOMs are formed. Finally, the pertinent low-order matrices (generated through this process) are added to $2^{p}$-SAOMs until the final $n$-SAOMs are constructed. The MCI is calculated by summing up the traces of all the $n$-SAOMs. Let $n=\sum_{i=0}^{p} a_{i} 2^{i}$ be the binomial representation of $n$. The number of steps in the algorithm is equal to $p+\sum_{i=0}^{p-1} a_{i}$. The strengh of the algorithm is twofold: (i) it avoids the repetition of 
partial matrix multiplications by constructing the SAOMs in an efficient way and (ii) a screening procedure is applied to every step of the process so that the number of SAOMs at each step is reduced. The algorithm could be enhanced by the use of an orbital localization scheme to increase the sparsity of the SAOMs in eqn (12).

\section{New electronic aromaticity index}

Despite the speed up gained by the algorithm described in the last section, we anticipate that the calculation of MCI on nonplanar rings of more than 16 members still poses a serious challenge. Moreover, for such large rings the numerical error in MCI and $I_{\text {ring }}$ is quite big. For these reasons, we prefer to explore the possibility of using other electronic mesures to account for the aromaticity of large rings. The FLU has been successfully used in the past to study the ground-state structure of several porphyrins. ${ }^{18}$ However, we have discussed the limitations of reference-based indices such as FLU to account for the aromaticity in molecules with stretched bonds or to recognize the aromaticity of transition state structures. ${ }^{13,25}$

In a previous work ${ }^{57}$ we studied the long-range delocalization patterns in annulenes and found that the interactions separated by an even number of atoms (e.g., 1,4 and 1,6) are larger than those separated by an odd number of atoms (1,3 and 1,5, respectively) in aromatic molecules. This finding is in line with the well-known fact that the meta-interaction is smaller than the para-interaction in benzene ${ }^{36}$ (and most aromatic six-membered rings ${ }^{38}$ ) despite the larger separation of the atoms in the para position. Since we want to capture multicenter delocalization effects, we suggest to average all the 4c-ESI values along the ring that keeps a positional relationship of $1,2,4,5$, as indicated in Fig. 1. This quantity (hereafter named AV1245), will be large if there is an important delocalization between bonds 1-2 and 4-5, a condition that only occurs in conjugated circuits. Note that the atom in position 3 is skipped intentionally because we want to include two 1-to-4 interactions $(1,4$ and 2,5$)$ and measure the extent of transferability of the delocalized electrons in the 1-2 bond to bond 4-5 and viceversa. We have explored other possibilities including these interactions (e.g. AV124) but none have given as good results as this one.

AV1245 does not rely on reference values, and it does not present any limitation on the nature of atoms, the molecular geometry or the level of calculation (we suggest to use eqn (11) with $a=1$ ). Besides, it does not suffer from large numerical precision errors, size-extensivity problems or unfavorable computational scaling.

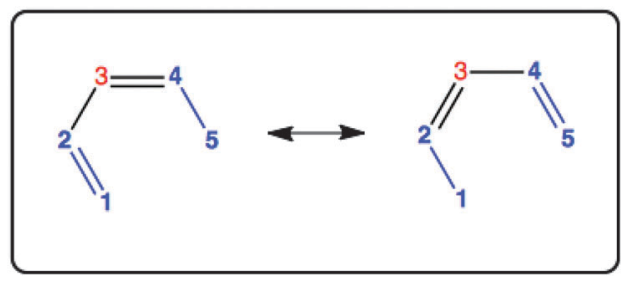

Fig. 1 AV1245 values for the six-membered rings.
AV1245 bears a very modest cost of $12 \mathrm{~m}^{3} n$ that thus only grows linearly with the ring size. The only limitation of AV1245 is that it cannot be applied to rings of less than six members. However, for such small rings we can safely use the MCI.

\section{Computational details}

We have studied several molecular systems that can be classified into five sets. The first set contains a series of neutral annulenes: benzene, $\mathrm{C}_{8} \mathrm{H}_{8}$ (planar), [10]annulene, [12]annulene, [14]annulene, [16]annulene, and [18]annulene, which will be used to assess the capabilities of the indices to recognize aromaticity as the number of $\pi$ electrons increases. The second set contains a series of molecules that are used to validate the performance of the new index in very different carbon-carbon bonds: cyclohexane, cyclohexene, benzene and the transition state of the Diels-Alder reaction occurring between butadiene and acetylene. A series of heteroaromatic compounds with six-membered rings (6-MR) will be used to guarantee a correct description of molecules containing heteroatoms: pyridine, pyridazine, pyrimidine, pyrazine, triazine, borazine and purine. The fourth set comprises some polycyclic aromatic hydrocarbons: naphthalene, anthracene, phenanthrene, benzocyclobutadiene, azulene and pentalene for which we analyze the 6-MRs and the peripheral rings. For the sake of completeness we have included two non-aromatic seven and eight-membered ring molecules $\left(\mathrm{C}_{7} \mathrm{H}_{14}\right.$ and $\left.\mathrm{C}_{8} \mathrm{H}_{16}\right)$, an aromatic seven-membered ring $\left(\mathrm{C}_{7} \mathrm{H}_{7}^{+}\right)$and hexaethynyl-carbo-benzene $\left(\mathrm{C}_{18} \mathrm{H}_{6}\right)$ as the molecule with a very large ring. ${ }^{58}$ This gives a total of seventeen 6-MRs, three 7-MRs, four 8-MRs, one 9-MR, four 10-MRs and one 12-MR (total 30 points) that will be used to compare the performance of MCI and AV1245. In addition, there are four 14-MRs, one 16-MR and two 18-MRs data for which only AV1245 values will be provided. We also include a family of [32] octaphyrins$(1,0,1,0,1,0,1,0)$ (see ref. 21 ) to analyze the performance of AV1245 in extended porphyrins.

The calculations have been performed using the g09 package $\mathrm{e}^{59}$ at the B3LYP/aug-cc-pVDZ level, except for octaphyrins that have been analyzed at the B3LYP/6-31G(d,p) level (LANL2DZ basis set and pseudopotential for Pd). ${ }^{21}$ The AOM calculations use the QTAIM partition and have been computed using the AIMall package ${ }^{60}$ and inputed into the ESI-3D code. ${ }^{61}$ The algorithm described in section 5 is used for the MCI values calculated in this work.

\section{Results}

In Fig. 2 we plot AV1245 against MCI for the 6-MR studied in this work. The data comprise aromatic and non-aromatic rings, the transition state of the Diels-Alder reaction (which is a difficult case for several aromaticity indices $)^{25}$ and the set of heteroaromatic molecules. AV1245 gives an excellent linear correlation with a zero intercept and a Pearson coefficient of $r^{2}=0.98$. The only small discrepancy between both indices is pyrazine which according to AV1245 is slightly more aromatic than benzene. 


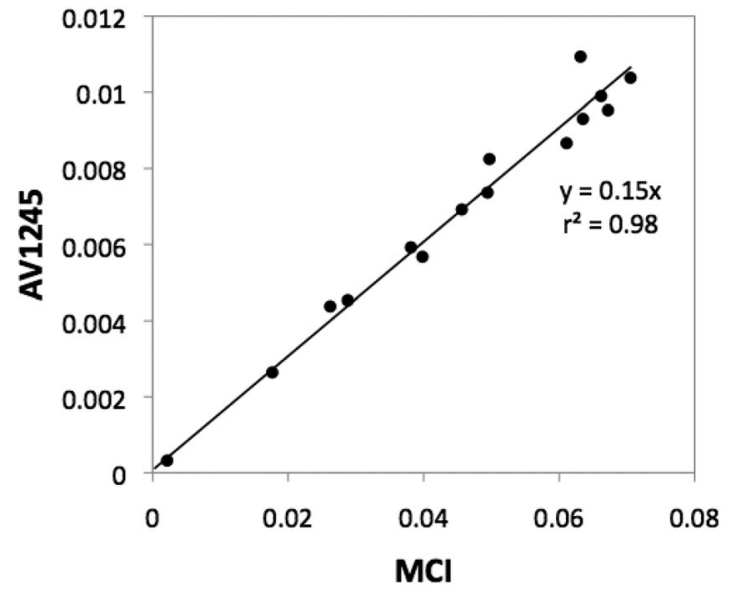

Fig. 2 AV1245 values for six-membered rings.

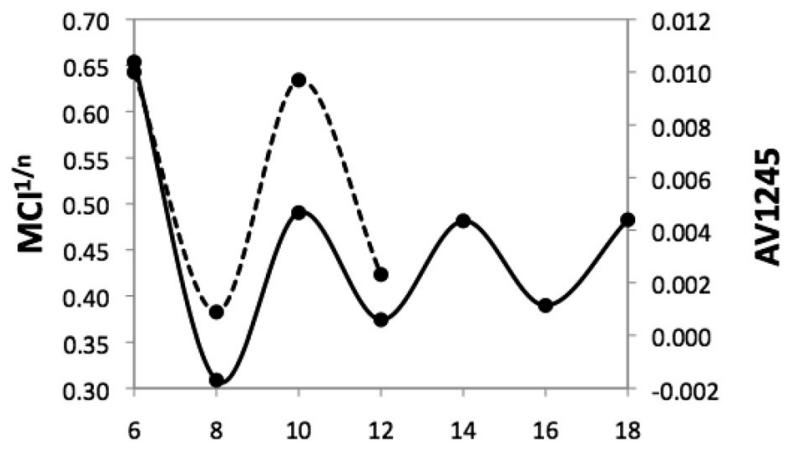

Fig. $3 \mathrm{MCl}^{1 / n}$ (dashed line) and AV1245 (solid line) for a series of annulenes $\mathrm{C}_{2 m} \mathrm{H}_{2 m}(m=3-9)$ as a function of the number of carbon atoms.

In Fig. 3 we plot AV1245 and $\mathrm{MCI}^{1 / n}$ against the number of carbon atoms for the series of neutral annulenes (set 1). The number of $\pi$ electrons is equal to the number of carbon atoms and, therefore, according to Hückel's rule, one expects a zig-zag plot where aromatic and antiaromatic compounds alternate. Although some antiaromatic compounds present negative MCI values, the experience indicates that many antiaromatic compounds simply give very small numbers and are barely distinguishable from non-aromatic compounds. ${ }^{15}$

Let us now analyze the ring-size dependency of both indices by including all rings from 6-MR to 12-MR in the plot of Fig. 4 . The indices do not show a linear correlation trend but, instead, a power-law dependency that fits most of the data points studied. A few exceptions have been marked in blue and red. The blue bullets indicate antiaromatic molecules that show negative values for both MCI and AV1245. One is thus deemed to conclude that, as it happens in the MCI, antiaromatic compounds exhibit either negative or very small AV1245 values. The red bullets correspond to molecules with an odd number of ring members. These molecules clearly deviate from the general trend and put forward that the conjugation mechanism in such rings is less obvious. The aromaticity in these compounds is severely underestimated by AV1245 and until further explorations are performed AV1245 should be used in rings of even number of atoms.

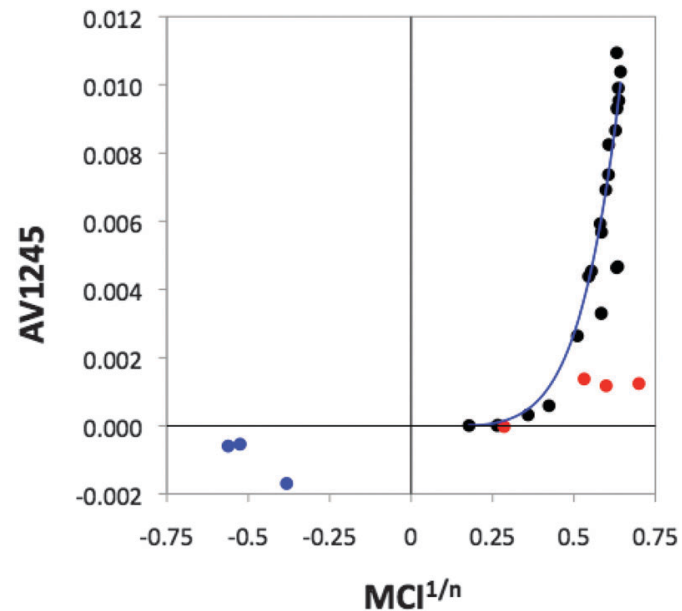

Fig. $4 \mathrm{AV} 1245 \mathrm{vs} . \mathrm{MCl}^{1 / n}$ for the whole series of rings up to twelve members. Red bullets indicate 7- and 9-membered-ring molecules and molecules with negative $\mathrm{MCl}$ values are marked in blue. The solid blue line corresponds to $y=0.1 \mathrm{MCl}^{5.2 / n}$.

In Table 2 we collect some AV1245 and $\mathrm{MCI}^{1 / n}$ values for large rings. Among the polycyclic aromatic hydrocarbons (PAHs), the most aromatic 6-MR is the external ring of phenanthrene, whose two-ring naphathalenic structure is the least aromatic 10-MR among PAHs. The peripheral ring of naphthalene shows a similar AV1245 value to the [10]annulene structure, whereas neither the peripheral ring of anthracene nor phenanthrene is as aromatic as [14]annulene. Conversely, hexaethynyl-carbobenzene also exhibits similar aromaticity to [18]annulene, which confirms the prominent aromatic character of this molecule. ${ }^{58}$ According to $\mathrm{MCI}^{1 / n}$ values the molecules are ordered in the same way as AV1245 for rings of the same size, thus showing the equivalence between both aromaticity measures. Comparisons of MCI and AV1245 among rings of different sizes do not provide the very same order due to the different ring-size dependency described above.

Finally, let us apply AV1245 to a series of extended porphyrins that can undergo a topological change between Hückel and

Table 2 AV1245 values (times 1000) and $\mathrm{MCl}^{1 / n}$ for selected rings

\begin{tabular}{|c|c|c|c|}
\hline & & AV1245 & $\mathrm{MCI}^{1 / n}$ \\
\hline 6-MR & Cyclohexane & 0.00 & 0.264 \\
\hline 6-MR & Benzene & 10.38 & 0.643 \\
\hline 6-MR & Naphthalene & 5.93 & 0.580 \\
\hline 6-MR & Anthracene ext. & 4.53 & 0.553 \\
\hline 6-MR & Anthracene int. & 4.38 & 0.545 \\
\hline 6-MR & Phenanthrene ext. & 6.92 & 0.598 \\
\hline 6-MR & Phenanthrene int. & 2.64 & 0.510 \\
\hline 10-MR & Naphthalene & 4.63 & 0.631 \\
\hline 10-MR & Anthracene & 3.76 & 0.603 \\
\hline 10-MR & Phenanthrene & 3.30 & 0.584 \\
\hline 14-MR & Anthracene & 3.51 & - \\
\hline 14-MR & Phenanthrene & 3.63 & - \\
\hline 10-MR & $\mathrm{C}_{10} \mathrm{H}_{10}$ & 4.66 & 0.634 \\
\hline 12-MR & $\mathrm{C}_{12} \mathrm{H}_{12}$ & 0.59 & 0.423 \\
\hline 14-MR & $\mathrm{C}_{14} \mathrm{H}_{14}$ & 4.35 & - \\
\hline 16-MR & $\mathrm{C}_{16} \mathrm{H}_{16}$ & 1.13 & - \\
\hline 18-MR & $\mathrm{C}_{18} \mathrm{H}_{18}$ & 4.39 & - \\
\hline 18-MR & $\mathrm{C}_{18} \mathrm{H}_{6}$ & 4.42 & - \\
\hline
\end{tabular}




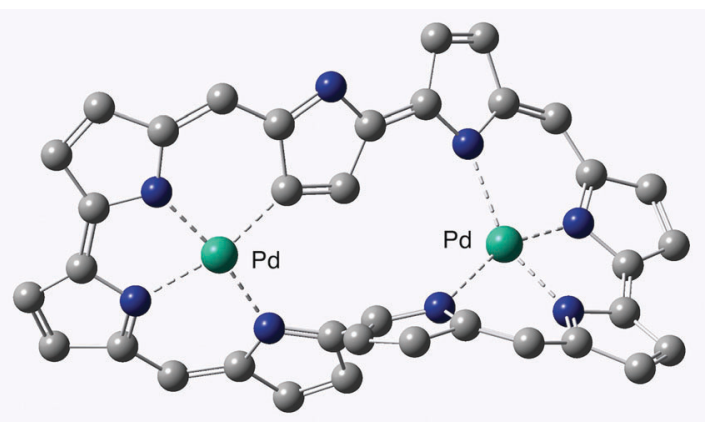

Fig. $52 \mathrm{M}$ Möbius-like intermediate in the isomerization path of Pd bismetalated [32]octaphyrin $(1,0,1,0,1,0)$; see ref. 21.

Table 3 AV1245 values (times 1000) for two 32-atom circuits of octaphyrins. The optimal circuit follows the conjugations of double-bonds (visiting only nitrogen atoms that were not originally protonated), whereas the non-optimal circuits go through nitrogen atoms that were protonated in the free-base form

\begin{tabular}{lll}
\hline & Optimal & Non-optimal \\
\hline $\mathrm{H}$ & 1.78 & 1.16 \\
$\mathrm{M}$ & 1.83 & 1.00 \\
$1 \mathrm{H}$ & 2.82 & 2.49 \\
$2 \mathrm{M}$ & 2.77 & 2.54 \\
$3 \mathrm{M}$ & 2.82 & 2.55 \\
$4 \mathrm{H}$ & 2.74 & 2.38 \\
$5 \mathrm{M}$ & 2.79 & 2.52 \\
\hline
\end{tabular}

Möbius isomers. To this aim, we have chosen free-base and Pd bis-metalated [32] octaphyrins $(1,0,1,0,1,0,1,0)$ involving Hückel and Möbius macrocyclic topologies. Namely, the free-base [32] octaphyrins $(1,0,1,0,1,0,1,0)$ Hückel $(\mathrm{H})$ and Möbius (M) conformers are compared against the five bis-metalated counterparts $(1 \mathrm{H}, 2 \mathrm{M}, 3 \mathrm{M}, 4 \mathrm{H}$ and $5 \mathrm{M})$ that enter the isomerization reaction path described by Ma and coworkers (see Fig. 5). ${ }^{21}$ The free-base [32] octaphyrins $(1,0,1,0,1,0,1,0)$ follow a 32-atom conjugated circuit that visits only the nitrogen atoms that are not protonated. One can also measure the aromaticity in the opposite circuit that only visits the protonated nitrogen atoms and, therefore, it is expected to be much less aromatic. The results in Table 3 show that the 32-atom conjugated circuits along the octaphyrin structures are most aromatic among free-base octaphyrins, whereas after metalation the difference between the two circuits is smaller. Nonetheless, the AV1245 values of free-base octaphyrins are quite small and, therefore, they could not be considered aromatic. Conversely, bis-metalated [32] octaphyrins $(1,0,1,0,1,0,1,0)$ are aromatic according to AV1245, and as expected from previous studies. ${ }^{21}$ Overall, the AV1245 values suggest that the aromaticity of these molecules is moderate and can be considered superior to the inner ring of phenanthrene (see Table 2).

\section{Conclusions}

We have introduced a new electronic aromaticity index, AV1245, consisting of an average of the 4-center MCI values along the ring that keep a positional relationship of 1, 2, 4, 5. AV1245 measures the extent of transferability of the delocalized electrons between bonds 1-2 and 4-5, which is expected to be large in conjugated circuits and, therefore, in aromatic molecules. AV1245 analyzes the aromaticity of individual rings (local aromaticity) but it could also be used to measure the extent of all 1, 2, 4, 5 conjugation interactions in a molecule and, therefore, account for the global aromaticity of annulated species such as benzenoid macrocycles. ${ }^{62}$ A new algorithm for the calculation of MCI for medium-sized rings has been introduced and used to produce the data for the calibration of the new aromaticity index.

Our results indicate that AV1245 correlates very well with MCI except for rings with an odd number of members. AV1245 does not rely on reference values, does not suffer from large numerical precision errors, and it does not present any limitation on the nature of atoms, the molecular geometry or the level of calculation. It is a size-extensive measure with a small computational cost that grows linearly with the number of ring members. Therefore, it is specially suited for studying the aromaticity of large molecular rings as those occurring in belt-shaped Möbius structures or porphyrins.

\section{Acknowledgements}

I am indebted to Dr Tomás Rocha for his kind hospitality during my stay in the Institute of Chemistry at the UNAM (Mexico), where this work was partially carried out. The author thanks Dr RamosCordoba and Dr Feixas for fruitful discussions. This research has been funded by Spanish MINECO Project No. CTQ2014-52525-P and the Basque Country Consolidated Group Project No. IT588-13. The author acknowledges the computational resources and technical and human support provided by SGI/IZO-SGIker UPV/EHU.

\section{References}

1 G. Frenking and A. Krapp, J. Comput. Chem., 2007, 28, 15-24.

2 R. Hoffmann, Am. Sci., 2015, 103, 18.

3 P. von Ragué Schleyer and H. Jiao, Pure Appl. Chem., 1996, 68, 209.

4 J. Poater, M. Duran, M. Solà and B. Silvi, Chem. Rev., 2005, 105, 3911-3947.

5 Z. Chen, C. S. Wannere, C. Corminboeuf, R. Puchta and P. v. R. Schleyer, Chem. Rev., 2005, 105, 3842-3888.

6 T. M. Krygowski, H. Szatylowicz, O. A. Stasyuk, J. Dominikowska and M. Palusiak, Chem. Rev., 2014, 114, 6383-6422.

7 F. Feixas, E. Matito, J. Poater and M. Solà, Chem. Soc. Rev., 2015, 44, 6389-6646.

8 A. R. Katritzky, P. Barczynski, G. Musumarra, D. Pisano and M. Szafran, J. Am. Chem. Soc., 1989, 111, 7.

9 X. Li, A. E. Kuznetsov, H.-F. Zhang, A. I. Boldyrev and L.-S. Wang, Science, 2001, 291, 859-861.

10 A. I. Boldyrev and L.-S. Wang, Chem. Rev., 2005, 105, 3716-3757.

11 R. Islas, T. Heine and G. Merino, Acc. Chem. Res., 2011, 45, 215-228.

12 F. Feixas, E. Matito, J. Poater and M. Solà, WIREs, Comput. Mol. Sci., 2013, 3, 105-122. 
13 F. Feixas, E. Matito, J. Poater and M. Solà, J. Comput. Chem., 2008, 29, 1543-1554.

14 F. Feixas, J. Jiménez-Halla, E. Matito, J. Poater and M. Solà, J. Chem. Theory Comput., 2010, 6, 1118-1130.

15 J. Cioslowski, E. Matito and M. Solà, J. Phys. Chem. A, 2007, 111, 6521-6525.

16 M. Alonso, B. Pinter, P. Geerlings and F. De Proft, Chem. - Eur. J., 2015, 21, 17631-17638.

17 A. Osuka and S. Saito, Chem. Commun., 2011, 47, 4330-4339.

18 F. Feixas, M. Solà and M. Swart, Can. J. Chem., 2009, 87, 1063-1073.

19 M.-C. Yoon, P. Kim, H. Yoo, S. Shimizu, T. Koide, S. Tokuji, S. Saito, A. Osuka and D. Kim, J. Phys. Chem. B, 2011, 115, 14928-14937.

20 E. Marcos, J. M. Anglada and M. Torrent-Sucarrat, J. Phys. Chem. C, 2012, 116, 24358-24366.

21 Z. Liu, Z. Tian, W. Li, S. Meng, L. Wang and J. Ma, J. Org. Chem., 2012, 77, 8124-8130.

22 L.-L. Li and E. W.-G. Diau, Chem. Soc. Rev., 2013, 42, 291-304.

23 E. Matito, M. Duran and M. Solà, J. Chem. Phys., 2005, 122, 014109.

24 J. Kruszewski and T. M. Krygowski, Tetrahedron Lett., 1972, 13, 3839-3842.

25 E. Matito, J. Poater, M. Duran and M. Solà, THEOCHEM, 2005, 727, 165.

26 P. v. R. Schleyer, C. Maerker, A. Dransfeld, H. Jiao and N. J. v. E. Hommes, J. Am. Chem. Soc., 1996, 118, 6317-6318.

27 P. Lazzeretti, Phys. Chem. Chem. Phys., 2004, 6, 217-223.

28 F. Feixas, M. Solà, J. M. Barroso, J. M. Ugalde and E. Matito, J. Chem. Theory Comput., 2014, 10, 3055-3065.

29 R. F. W. Bader and M. E. Stephens, Chem. Phys. Lett., 1974, 26, 445.

30 R. F. W. Bader and M. E. Stephens, J. Am. Chem. Soc., 1975, 97, 7391-7399.

31 X. Fradera, M. A. Austen and R. F. W. Bader, J. Phys. Chem. A, 1999, 103, 304-314.

32 E. Matito, M. Solà, P. Salvador and M. Duran, Faraday Discuss., 2007, 135, 325-345.

33 P.-O. Löwdin, Phys. Rev., 1955, 97, 1474-1489.

34 E. Matito, M. Duran and M. Solà, J. Chem. Phys., 2006, 125, 059901.

35 I. Mayer, Chem. Phys. Lett., 1983, 97, 270-274.

36 R. L. Fulton, J. Phys. Chem., 1993, 97, 7516-7529.

37 R. F. W. Bader, A. Streitwieser, A. Neuhaus, K. E. Laidig and P. Speers, J. Am. Chem. Soc., 1996, 118, 4959.

38 J. Poater, X. Fradera, M. Duran and M. Solà, Chem. - Eur. J., 2003, 9, 400-406.

39 M. Giambiagi, M. S. de Giambiagi and K. C. Mundim, Struct. Chem., 1990, 1, 423-427.

40 M. Giambiagi, M. S. de Giambiagi, C. D. dos Santos Silva and A. P. de Figuereido, Phys. Chem. Chem. Phys., 2000, 2, 3381-3392.

41 P. Bultinck, R. Ponec and S. Van Damme, J. Phys. Org. Chem., 2005, 18, 706-718.

42 R. Ponec and I. Mayer, J. Phys. Chem. A, 1997, 101, 1738-1741.

43 A. B. Sannigrahi and T. Kar, Chem. Phys. Lett., 1999, 299, 518-526.
44 E. Francisco, A. M. Pendás and M. A. Blanco, J. Chem. Phys., 2007, 126, 094102.

45 A. C. Castro, E. Osorio, J. L. Cabellos, E. Cerpa, E. Matito, M. Solà, M. Swart and G. Merino, Chem. - Eur. J., 2014, 20, 4583-4590.

46 I. Gutman, M. Milun and N. Trinajstić, J. Am. Chem. Soc., 1977, 99, 1692.

47 W. Heyndrickx, P. Salvador, P. Bultinck, M. Solà and E. Matito, J. Comput. Chem., 2011, 32, 386-395.

48 E. Matito, M. Solà, M. Duran and P. Salvador, J. Phys. Chem. A, 2006, 110, 5108-5113.

49 E. Matito, J. Poater, M. Solà, M. Duran and P. Salvador, J. Phys. Chem. A, 2005, 109, 9904-9910.

50 M. Rodríguez-Mayorga, E. Ramos-Cordoba, P. Salvador, M. Solà and E. Matito, Mol. Phys., DOI: 10.1080/00268976.2015.1121297.

51 R. F. W. Bader, Atoms in Molecules: A Quantum Theory, Oxford Univ. Press, Oxford, 1990.

52 P. Bultinck, M. Mandado and R. Mosquera, J. Math. Chem., 2008, 43, 111-118.

53 P. W. Fowler and E. Steiner, Chem. Phys. Lett., 2002, 364, 259-266.

54 L. Lain, A. Torre and R. Bochicchio, J. Phys. Chem. A, 2004, 108, 4132-4137.

55 F. Feixas, M. Rodrguez-Mayorga, E. Matito and M. Solà, Comput. Theor. Chem., 2015, 1053, 173-179.

56 J. M. Mercero, E. Matito, F. Ruipérez, I. Infante, X. Lopez and J. M. Ugalde, Chem. - Eur. J., 2015, 21, 9610-9614.

57 F. Feixas, E. Matito, M. Solà and J. Poater, Phys. Chem. Chem. Phys., 2010, 12, 7126-7137.

58 C. Lepetit, C. Godard and R. Chauvin, New J. Chem., 2001, 25, 572-580.

59 M. J. Frisch, G. W. Trucks, H. B. Schlegel, G. E. Scuseria, M. A. Robb, J. R. Cheeseman, G. Scalmani, V. Barone, B. Mennucci, G. A. Petersson, H. Nakatsuji, M. Caricato, X. Li, H. P. Hratchian, A. F. Izmaylov, J. Bloino, G. Zheng, J. L. Sonnenberg, M. Hada, M. Ehara, K. Toyota, R. Fukuda, J. Hasegawa, M. Ishida, T. Nakajima, Y. Honda, O. Kitao, H. Nakai, T. Vreven, J. A. Montgomery, Jr., J. E. Peralta, F. Ogliaro, M. Bearpark, J. J. Heyd, E. Brothers, K. N. Kudin, V. N. Staroverov, R. Kobayashi, J. Normand, K. Raghavachari, A. Rendell, J. C. Burant, S. S. Iyengar, J. Tomasi, M. Cossi, N. Rega, J. M. Millam, M. Klene, J. E. Knox, J. B. Cross, V. Bakken, C. Adamo, J. Jaramillo, R. Gomperts, R. E. Stratmann, O. Yazyev, A. J. Austin, R. Cammi, C. Pomelli, J. W. Ochterski, R. L. Martin, K. Morokuma, V. G. Zakrzewski, G. A. Voth, P. Salvador, J. J. Dannenberg, S. Dapprich, A. D. Daniels, Ö. Farkas, J. B. Foresman, J. V. Ortiz, J. Cioslowski and D. J. Fox, Gaussian 09 Revision D.01, Gaussian Inc., Wallingford CT, 2009.

60 T. A. Keith, AIMAll (Version 14.11.23), TK Gristmill Software, Overland Park KS, USA, 2014, aim.tkgristmill.com.

61 E. Matito, ESI-3D: Electron Sharing Indices Program for $3 D$ Molecular Space Partitioning, Institute of Computational Chemistry and Catalysis, University of Girona, Catalonia, Spain, 2015.

62 E. Matito, F. Feixas and M. Solà, THEOCHEM, 2007, 811, 3. 